\section{Apoyo social y condiciones de vida de adultos mayores que viven en la pobreza urbana en México}

\author{
Social support and living conditions in \\ poor elderly people in urban Mexico
}

Blanca Estela Pelcastre-Villafuerte 1

Sandra Treviño-Siller 1

Tonatiuh González-Vázquez ${ }^{1}$

Margarita Márquez-Serrano 1
1 Instituto Nacional de Salud Pública, Cuernavaca, México.

Correspondencia

M. Márquez-Serrano

Instituto Nacional de Salud Pública.

Av. Universidad 655,

Cuernavaca, Morelos,

62100, México.

mmarquez@correo.insp.mx

\section{Abstract}

The aim of this paper was to analyze social support and living conditions among poor elderly people in Mexican cities. A qualitative study with eight focus groups was carried out in Guadalajara, Cuernavaca, Chilpancingo, and Culiacan, Mexico, in 2005. Forty men and 63 women participated in the study. The main support for the elderly in daily living came from their immediate family and in some cases from neighbors. Social support was basically material and economic, in addition to providing company and transportation for medical appointments. Daily emotional support, companionship, and social inclusion were minimal or absent. The study identified a significant lack of support from government and religious or civil society organizations. The family is still the main source of support for the elderly. Increased government collaboration is dramatically needed to combat the misconception that the needs of the elderly are the individual family's responsibility rather than a collaborative effort by society.

Aged; Poverty; Social Conditions; Social Support

\section{Introducción}

Actualmente se vive una transición demográfica mundial nunca antes vista, la distribución de la población entre los distintos grupos de edad ha experimentado una serie de cambios, evidenciando un progresivo envejecimiento. En el 2050, según las proyecciones para México, la población mayor de 60 años será igual o superior que la de menores de 15 años 1 , lo que significa que dicha población se incrementará de los poco más de 9,6 millones que existían en 20062 a entre 27 y 32 millones 3 ; además, la mayor parte residirá en zonas urbanas empobrecidas 4.

La vulnerabilidad social es la desprotección de un grupo social cuando enfrenta daños potenciales a su salud, amenazas a la satisfacción de sus necesidades y violación a sus derechos humanos por no contar con recursos personales, sociales y legales 5. En la actualidad el 70\% de adultos mayores vive en municipios de bajo o muy bajo desarrollo social, con una mayor desprotección y vulnerabilidad en comparación con otros grupos poblacionales ${ }^{6}$. Estos cambios demográficos representan importantes retos, debido a que el gobierno mexicano no ha desarrollado ni la infraestructura necesaria, ni la capacidad humana para atender las demandas y garantizar el bienestar de los adultos mayores, ni tampoco para enfrentar el incremento de pobreza en el país, debido a la reciente crisis económica. 
En países de ingresos medios como México, los apoyos institucionales como la seguridad social o la cobertura de servicios de salud son escasos, además de que a los ahora adultos mayores les faltó acceso a recursos financieros durante su juventud, con los que pudieron haber acumulado fondos para enfrentar la vejez 7; por ejemplo, en el año 2005 el $44 \%$ de los ancianos no contaba con derechohabiencia a servicios de salud ${ }^{2}$. Ante este escenario cobran importancia actores sociales cercanos que apoyan cotidianamente y en situaciones de emergencia a los adultos mayores.

En los últimos años diversos autores 8,9,10 han estudiado la existencia y el efecto de los apoyos sociales, especialmente en el caso de los adultos mayores, pues constituyen un grupo vulnerable que dependiendo de sus propios recursos, grado de funcionalidad, así como de su condición de género, económica y de salud, es mayor o menormente apoyado por el grupo familiar o social más cercano. Además, debido al poco apoyo de la mayoría de los gobiernos y a los recursos públicos insuficientes asignados a este grupo de población en rápido crecimiento, el estudio y la comprensión de cómo sobreviven los adultos mayores, especialmente en situación de pobreza, se convierte en un tema relevante 11,12,13,14,15.

En diversas reuniones internacionales se ha considerado el tema de los apoyos y las redes sociales como prioritario en el estudio y definición de políticas para el envejecimiento 16,17 . Este tipo de estudios han sido más frecuentes en países desarrollados y recientemente se han comenzado a realizar en algunos países latinoamericanos y en México 18.

Ya desde la segunda mitad de los 70, autores como Cassel 19 y Cobb 20 propusieron que los apoyos sociales juegan un papel central en el mantenimiento de la condición de salud y bienestar de los individuos, independientemente del modelo utilizado para explicar la asociación entre apoyo social y condición de salud (efecto directo o efecto amortiguador), diversos estudios han identificado que el tipo de apoyos con que se cuenta está asociado a diferentes estados de salud y bienestar 8,10, aún en individuos que se encuentran en situaciones de similar vulnerabilidad 21,22.

Tal y como comentan Barrón \& Sánchez-Moreno 23 , no hay una definición clara y consensuada de apoyo social, no obstante la cantidad de estudios publicados en las últimas décadas. Según Matud et al. 24, los apoyos sociales se pueden definir como todas aquellas acciones de diversa índole (emocional, instrumental e informacional 24 , cognitiva y material 18 ), que son realizadas por diferentes actores con el propósito fundamental de contribuir al bienestar de otro en una situación cotidiana o de emergencia. De acuerdo a Khan \& Antonucci (1980, citados por Guzmán et al. 18), los apoyos sociales son las transacciones interpersonales que implican ayuda, afecto y afirmación.

Es importante resaltar que estos apoyos se dan en el contexto de una red social previamente establecida entre un conjunto de personas. Diversos estudios han reportado que la existencia y calidad de estos apoyos sociales están relacionadas con una mejor experiencia de vida y con mejores condiciones de salud tanto en población general, como en población adulta mayor 15,18,24.

La familia, a pesar de su proceso de transformación estructural 25 , sigue siendo la base de los apoyos sociales, al representar el grupo social inicial del individuo. En algunos estudios se muestra que en muchas ocasiones los adultos mayores pobres no tienen mayor opción que vivir con los hijos o que, por el contrario, son ellos quienes proveen de recursos a la descendencia, bien sea por jubilación o por incentivos recibidos de diferentes programas gubernamentales y de la sociedad civil 15. Es importante estudiar cómo están resolviendo actualmente los adultos mayores su vida cotidiana, sobre todo cuando sabemos que la composición familiar en nuestro país, como en la mayoría de los países latinoamericanos, está transitando del modelo de familia extensa al de familia nuclear, lo cual implica menos miembros adultos que pueden prestar apoyo a los más vulnerables 15,25 .

El presente artículo tiene como objetivo describir y analizar las características principales de los apoyos sociales, formales e informales, de los adultos mayores que viven en la pobreza urbana relacionándolos con las condiciones de vida y vivencia del envejecimiento. Entendiendo por apoyos formales, todos los ingresos económicos generados por los propios adultos mayores a través de algún trabajo remunerado y la transferencia de recursos del ámbito formal (sistema de salud y seguridad social) y por apoyos informales, los transferidos por la familia, vecinos, red de amistades y/o por la comunidad 26 .

\section{Metodología}

Se diseñó un estudio cualitativo y se trabajó mediante la técnica de grupos focales con adultos mayores (hombres y mujeres) durante 20052006. Para el desarrollo del estudio se seleccionaron cuatro ciudades correspondientes a cuatro diferentes regiones del país: norte (Culiacán, Sinaloa), occidente (Guadalajara, Jalisco), centro (Cuernavaca, Morelos) y sur (Chilpancingo, Guerrero). 
La selección de colonias se llevó a cabo utilizando los indicadores municipales de marginación del Consejo Nacional de Población (CONAPO); posteriormente se hizo una visita de reconocimiento de campo para asegurar que las colonias en las cuales se levantarían los datos se encontraran dentro de la zona metropolitana y que tuvieran las siguientes características a la inspección visual: calles sin pavimentar, casas construidas de materiales precarios y servicio deficiente de electricidad, agua potable y drenaje.

Se organizaron 8 grupos focales, uno de varones y uno de mujeres en cada ciudad, buscando un promedio de 6 a 8 participantes por grupo 27 .

Los criterios de selección fueron: ser mayor de 60 años, estar en condiciones de participar activamente en una sesión (escuchar y hablar bien) y aceptar la grabación de las sesiones. Todos fueron informados sobre el objetivo del estudio, la dinámica de las sesiones y sus derechos como participantes, además de firmar una carta de consentimiento informado, previamente aprobada por el Comité de Ética del Instituto Nacional de Salud Pública.

Se diseñó una guía específica para los grupos focales que abordó los siguientes temas: perfil de los participantes, lazos familiares y sociales, condiciones generales de salud, apoyo institucional, percepción de bienestar, significado de la vejez y propuestas para mejorar la vivencia del envejecimiento. El total de participantes fue de 103 (40 varones y 63 mujeres). La duración promedio de los grupos focales fue de dos horas.

Distintos contactos locales en cada ciudad ayudaron con la identificación de un lugar adecuado para el desarrollo de los grupos focales y en la invitación de los participantes. Todos los grupos fueron moderados por una de las investigadoras del equipo con amplia experiencia en esta técnica, quien siguió la guía específicamente diseñada para el estudio. De acuerdo al protocolo de la técnica 27 , todos los grupos iniciaron con una sesión de presentación del estudio y del equipo de trabajo dejando claro el objetivo de la investigación, seguido por una ronda de presentación de los participantes, lo que permitió iniciar la reflexión sobre el tema. La moderadora fue poniendo sobre la mesa cada uno de los puntos de la guía permitiendo la intervención de los participantes.

Bajo la perspectiva fenomenológica 28 se revisaron todos los registros de los grupos focales $\mathrm{y}$ se realizó un análisis de discurso por género y por ciudad. De acuerdo con Fairclough 29, definimos el discurso como una práctica social, en este sentido lo entendemos como lenguaje en uso que promueve ciertas relaciones sociales 30; para el análisis se definieron categorías analíticas (per- fil de los informantes, lazos familiares y sociales, percepción de bienestar, condiciones de salud, significados de la vejez y apoyo institucional) a partir de las cuales se organizó la información, se hizo una lectura crítica tratando de identificar recurrencias y diferencias en el discurso y tratando de encontrar vínculos entre las categorías, para finalmente integrar una descripción densa de cada categoría.

\section{Resultados}

\section{Perfil de los informantes}

Los participantes se caracterizaron por tener una edad promedio de 70,5 años, siendo más jóvenes (67 años) en Guadalajara y de mayor edad (74 años) en Chilpancingo; además, en todas las ciudades los varones eran mayores que las mujeres. Casi el 60\% carecía de escolaridad, siendo mayor en las mujeres y más de dos quintas partes no contaban con derechohabiencia (derecho a atención a salud en instituciones públicas), en su gran mayoría mujeres. La condición de viudez fue frecuente, lo cual se observó en más de un tercio de los participantes casi en su totalidad mujeres; sin embargo, únicamente vivían solos una cuarta parte del total de los adultos mayores, la mayoría vivía con el cónyuge en combinación con una variada gama de familiares (Tabla 1 ).

Lo más común fue que los informantes percibieran un estado de salud deteriorado, principalmente debido a las condiciones de vida previas y actuales, como por ejemplo, inicio en el trabajo a temprana edad, empleos que demandan un enorme desgaste físico, alimentación deficiente en algunos casos y malas condiciones de la vivienda. Es importante mencionar que los adultos mayores que vivían acompañados refirieron una mejor experiencia de envejecimiento, en contraste con aquellos que vivían solos.

\section{¿Quién brinda apoyo y qué tipo de apoyos se brindan?}

Entre los principales rasgos estructurales de la red social de estos adultos mayores se identificó: la co-residencia con la familia nuclear y en promedio tres miembros en el hogar. En términos generales se observó un intercambio de apoyos entre los adultos mayores y sus familiares cercanos, ellos reciben algún tipo de apoyo al mismo tiempo que se lo proporcionan a sus hijos y nietos. Las principales necesidades que los adultos mayores expresaron fueron las de techo, alimentación y acompañamiento y traslado a consultas o estudios médicos; sin embargo, a lo largo de 
Perfil sociodemográfico de los participantes. Cuernavaca, Chilpancingo, Culiacán y Guadalajara, México, 2005.

\begin{tabular}{|c|c|c|c|c|c|c|c|c|}
\hline & \multicolumn{2}{|c|}{ Cuernavaca } & \multicolumn{2}{|c|}{ Chilpancingo } & \multicolumn{2}{|c|}{ Culiacán } & \multicolumn{2}{|c|}{ Guadalajara } \\
\hline & Hombres & Mujeres & Hombres & Mujeres & Hombres & Mujeres & Hombres & Mujeres \\
\hline \multicolumn{9}{|l|}{ Características participantes } \\
\hline Total (n) & 10 & 20 & 9 & 14 & 14 & 17 & 9 & 12 \\
\hline \multirow[t]{2}{*}{ Edad promedio (años) } & 69 & 72 & 76 & 72 & 72 & 69 & 68 & 66 \\
\hline & $\%$ & $\%$ & $\%$ & $\%$ & $\%$ & $\%$ & $\%$ & $\%$ \\
\hline \multicolumn{9}{|l|}{ Estado civil } \\
\hline Soltera/o & - & 5,0 & - & 7,1 & - & - & 11,1 & - \\
\hline Casada/o & 40,0 & 35,0 & 66,6 & 42,8 & 78,5 & 29,4 & 55,5 & 33,3 \\
\hline Unión libre & 30,0 & 40,0 & 11,1 & - & - & - & - & 8,3 \\
\hline Viuda/o & - & - & 22,2 & 50,0 & 7,1 & 58,8 & 22,2 & 50,0 \\
\hline Separada/o & - & 20,0 & - & - & 14,2 & 11,7 & - & 8,3 \\
\hline Abandonada/o & 10,0 & - & - & - & - & - & 11,1 & - \\
\hline Sin información & 20,0 & - & - & - & - & - & - & - \\
\hline \multicolumn{9}{|l|}{ Escolaridad } \\
\hline Sin escolaridad & 50,0 & 55,0 & 66,6 & 85,7 & 71,4 & 47 & 44,4 & 33,3 \\
\hline Primaria incompleta & 40,0 & 25,0 & - & 7,1 & 21,4 & 29,4 & 44,4 & 66,6 \\
\hline Primaria completa & 10,0 & 15,0 & 22,2 & 7,1 & - & 5,8 & 11,1 & - \\
\hline Secundaria & - & 5,0 & - & - & 7,1 & - & - & - \\
\hline Normal superior & - & - & 11,1 & - & - & 17,6 & - & - \\
\hline \multicolumn{9}{|l|}{ Residencia } \\
\hline Con esposa/o & 20,0 & 15,0 & - & - & 14,2 & 17,6 & 11,1 & 25,0 \\
\hline Con esposa/o e hijos & 30,0 & 10,0 & - & 14,2 & 35,7 & 5,8 & 11,1 & 8,3 \\
\hline Con esposa/o y nieto & - & - & - & - & 7,1 & - & 11,1 & 8,3 \\
\hline Con hija/os & 10,0 & 25,0 & - & 35,7 & 28,5 & 29,4 & - & 25,0 \\
\hline Con nietos & - & 15,0 & 66,6 & 7,1 & - & 11,7 & - & 8,3 \\
\hline Con hija y nietos & - & 10,0 & 22,2 & - & - & - & - & - \\
\hline Con esposa/o, hija, yerno y nietos & 10,0 & 5,0 & 11,1 & - & 7,1 & - & 22,2 & - \\
\hline Con otros familiares & - & 5,0 & - & - & - & - & - & - \\
\hline Solo & 30,0 & 15,0 & - & 42,8 & 7,1 & 35,2 & 44,4 & 25,0 \\
\hline \multicolumn{9}{|l|}{ Derechohabiencia } \\
\hline Sí & 50,0 & 40,0 & 55,5 & 35,7 & 71,4 & 58,8 & 88,8 & 75,0 \\
\hline Ninguna & 50,0 & 60,0 & 44,4 & 64.2 & 28,5 & 411 & 11,1 & 25,0 \\
\hline
\end{tabular}

Fuente: elaboración propia con base en grupos focales con adultos mayores en Cuernavaca, Chilpancingo, Guadalajara y Culiacán, 2005.

las sesiones se hicieron constantes menciones de sentimientos de soledad, abandono y falta de integración social constante, tanto en hombres como en mujeres.

Una diferencia de género fue que los hombres resaltaron recurrentemente la importancia de tener una mujer a su lado en esta etapa de la vida: “...solo no, porque uno ya está impuesto (sic.), acostumbrado a estar acompañado, el que está solo, ya ni modo..."; "es que la mujer es como la segunda madre, los hijos menos, es la mujer la que cuida...". Chilpancingo fue la única ciudad donde los informantes refirieron un apoyo mutuo entre los cónyuges quienes compartían actividades o se dividían tareas para poder salir adelante.

Independientemente del género, se identificó que, después del cónyuge, el principal apoyo social proviene de los familiares cercanos sobre todo hijos, yernos, nueras, nietos y en menor medida, de los vecinos. El apoyo identificado en los ancianos es fundamentalmente de tipo material (sus familiares y/o vecinos les compran, llevan o preparan sus alimentos y/o medicamentos), económico (les dan dinero periódica u ocasionalmente o pagan consultas y tratamientos médicos) y de acompañamiento en eventos médicos 
(traslado a sus citas y tratamientos). En mucho menor medida se identificó el acompañamiento cotidiano (estar en casa con ellos, platicar y convivir) y la inserción social (participación en actividades sociales o laborales).

La relación con los familiares: caracterización de los vínculos

A pesar de que, en general, las relaciones familiares en esta etapa de la vida son muy importantes, en este estudio se identificó que no siempre se mantiene una buena relación con los hijos, nueras y nietos por lo que los adultos mayores consideran que es mejor mantener cierta distancia para evitar problemas. Esta percepción fue más común entre las mujeres con mejores condiciones de salud, con menor edad en comparación con los varones y con mayor posibilidad de funcionalidad e independencia.

Las mujeres participantes consideraron que el acercamiento de los hijos con los padres "depende mucho de cómo los hayan tratado cuando ellos eran pequeños" (mujer, Cuernavaca). Los hombres señalaron que: "Los hijos están más apegados con las madres, porque ellas fueron quienes los cuidaron de pequeños" (varón, Cuernavaca). Los adultos mayores esperan mucho de los hijos pues ellos consideran que "deben cuidarlos y atenderlos por todo lo que ellos, como padres, hicieron en su momento" (varón, Chilpancingo).

También reconocieron que el estado de salud, como la movilidad física, determina en gran parte las relaciones que se establecen con la gente que vive cerca, señalando que “...es más fácil vivir sola o únicamente con el cónyuge mientras una no esté tan mal de salud", pero que se va necesitando mayor compañía a medida que aparecen más enfermedades: "yo vivo con quien sea, a veces con una hija, a veces con una nieta, yo lo prefiero porque ya no puedo hacer nada por la reuma y la artritis, estoy mala de mis manos..." (mujer, Culiacán).

En Guadalajara, en los casos en donde algún familiar migró fuera del país, se señaló que el vínculo con otros familiares debe intensificarse para compensar la falta de este miembro dentro del hogar, pues: "los que se van al norte se olvidan de sus obligaciones...”.

Adicionalmente, durante la realización del grupo focal con los varones, se evidenció la necesidad de un vínculo que no se había considerado y es el que se puede establecer entre los propios adultos mayores, en este grupo en especial se dieron muestras de apoyo mutuo que en ningún otro grupo se observó, parecían requerir sólo el espacio y el momento adecuado para que estos ofrecimientos fluyeran. Inclusive, también en el grupo de mujeres de Cuernavaca se sugirió que tener una sesión mensual o quincenal similar a la desarrollada, serviría de mucho para tener un espacio en el cual compartir sus experiencias, problemas y necesidades con gente de su misma edad.

En relación a los tipos de apoyo los varones manifestaron que "hay hijos buenos y malos", pero lo común fue que afirmaran que sí los apoyan cuando se enferman, les dan dinero o los acompañan a sus consultas médicas. Tal parece que el apoyo más común con el que cuentan es para situaciones ocasionales en que se percibe la vulnerabilidad del adulto mayor y el acompañamiento de un miembro de la familia con mayor movilidad, ingresos y habilidades sociales se hace eminente.

En algunos casos los nietos de mayor edad, aparecen como apoyos (soporte económico y relativo acompañamiento), ya que si son muy pequeños más bien su cuidado es responsabilidad de los adultos mayores, situación más frecuente entre las mujeres, quienes con los nietos siguen desempeñando su rol tradicional de cuidadoras.

En los varones, las vecinas son importantes como actores sociales de apoyo, tanto en el caso de la alimentación, como de enfermedad: " $a$ veces los vecinos ayudan más que los hijos (...) dan cosas de despensa..."; para las mujeres el apoyo vecinal cumple más el rol de vigilancia de las viviendas o apoyo en caso de enfermedad. En reciprocidad el adulto mayor trata de llevarse bien con la familia vecina y colaborar con pequeñas tareas en esa casa.

\section{El apoyo mutuo: los adultos mayores y la familia}

Los adultos mayores reciben apoyo de la familia, pero también hacen aportaciones a ella. Los varones recurrentemente manifestaron su preocupación por no poder continuar trabajando y proveyendo económicamente. Esta situación se traduce en la imposibilidad de exigir, lo cual los hace sentirse obligados a "hacer algo a cambio, para tener derecho de vivir ahî".

Las mujeres siguen desarrollando diversas actividades domésticas como la limpieza de la casa. Pocas de ellas pueden lavar ropa o planchar debido a padecimientos que consideran propios de su edad, como artritis. También cuidan o recogen de la escuela a los nietos; aunque esta última tarea suele resultar pesada, la aceptan porque sus hijos trabajan y ellas como abuelas se sienten “obligadas" a apoyar. Algunas consideraron que ellas dan más ayuda de la que reciben por parte de sus hijos. 
Los varones que viven en casa de los hijos también realizan diversas actividades en el hogar como lavar el patio, barrer y arreglar descomposturas, incluso los que viven en pareja manifestaron colaborar con algunas tareas específicas (limpieza doméstica o hacer compras), más aún cuando las esposas están enfermas. Asimismo, con frecuencia se identificó que ellos cuidan de los nietos, les dan de comer, los recogen de la escuela, etc.; y que de esta manera se sienten merecedores de apoyo, comida o casa, pero sin derecho a intervenir en caso de conflictos familiares (Tabla 2).

\section{Y cómo responden las instituciones}

De acuerdo al discurso de los participantes, existe una ausencia de apoyo por parte de organizaciones de la sociedad civil para los adultos mayores, y el otorgado por el gobierno y la iglesia se consideró como insuficiente. Las mujeres de todos los estados mencionaron que el programa Oportunidades del gobierno federal (que busca disminuir los índices de pobreza extrema en México y que incluye un componente educativo, uno de salud y uno de nutrición), estaba enfocado a los nietos y que era cuestión de suerte obtenerlo, porque no siempre se apoya a quien más lo necesita. Sin embargo, existen particularidades de programas o acciones diferentes identificadas en cada ciudad. En Cuernavaca se reportó la entrega de productos alimenticios por parte de un programa de la iglesia católica conocido como "Por Cristo", y en Chilpancingo se señaló la desaparición de un programa de alimentos del Sistema para el Desarrollo Integral de la Familia (DIF) estatal, debido a que la ayuda la recibían no sólo las personas empobrecidas, sino muchas otras, como algunos empleados de gobierno.

Varias de las participantes de Cuernavaca y Chilpancingo, hablaron de la "Pensión Guerrero" (programa gubernamental para los adultos mayores que consiste en una ayuda bimestral de 800 pesos [64 USD]), cuya periodicidad y monto no fue fácilmente identificado por ellos. Las personas de Cuernavaca sugirieron que esto podría retomarse en su estado para apoyar verdaderamente a los adultos mayores.

Las mujeres de Chilpancingo señalaron que otro tipo de apoyo son los asilos públicos: "hay un asilo para ancianas, ahí las van a dejar sus familiares cuando ya no pueden con ellas o cuando ya son un estorbo, una carga". Este asilo es por parte del gobierno, es gratuito y "sí están bien atendidas, aunque falta atención médica y a veces hay problemas de alimentación”. Se comentó que cada vez más familias se avergüenzan de sus adultos mayores "son como un mueble vie- jo" y el gobierno "se hace sordo" y no escucha las demandas y necesidades de esta población. Los varones de Chilpancingo señalaron que los partidos políticos siempre les prometen muchas cosas en época de elecciones, pero que después no cumplen, manifestaron que el único programa exclusivo para los adultos mayores era la Pensión Guerrero, el cual, sin embargo, no se los dan a todos.

De los otros programas identificados como potenciales fuentes de apoyo (Oportunidades y Seguro Popular), comentaron que ninguno es exclusivo para los adultos mayores, sino que son intervenciones para población pobre. También se señaló la necesidad de apoyo en el transporte público en las ciudades, así como con el problema de la falta de empleo para los adultos mayores y la percepción de "no poder mantener a la familia" por esta situación.

En comparación con las otras ciudades, las mujeres y los hombres de Culiacán identificaron varios apoyos, pero aún así consideraron que son insuficientes para cubrir sus necesidades. Mencionaron el DIF, la organización religiosa Cáritas y otras iglesias con la repartición de alimentos; algunos descuentos ocasionales por parte del partido político gobernante y el programa Oportunidades.

En Guadalajara se comentó que se instituyó el "Día del Anciano" y por tal motivo se les hizo un desayuno. Hablaron del voluntariado del DIF y de la jubilación que pueden tener en caso de haber trabajado en una empresa formal, pero “trabajas muchos años y al final no tienes nada...”. Algunas de las personas entrevistadas opinaron que lo que en realidad haría falta es un programa de empleo para los adultos mayores.

Los varones reconocieron la existencia del programa Oportunidades y recomendaron la implementación de comedores para los adultos mayores necesitados. Si bien reconocieron la ayuda alimenticia del voluntariado del DIF, comentaron que han prometido alimentos gratuitos para ellos, pero que esto aún no se cumple. Adicionalmente, en todas las ciudades, se reconoció la existencia del Instituto Nacional para las Personas Adultas Mayores (INAPAM) que subsidia el $50 \%$ en el transporte foráneo.

\section{Discusión y conclusiones}

Tal como han reportado diversos autores 15,25, la familia continúa siendo la fuente fundamental de apoyo para los adultos mayores. Condiciones como la migración, la actividad laboral de los hijos (especialmente de las hijas y el necesario cuidado de los nietos), el vivir solos y la situa- 
Apoyos familiares otorgados por los adultos mayores del estudio.

\begin{tabular}{|c|c|c|c|c|}
\hline & \multicolumn{2}{|c|}{ Responsabilidades en el hogar } & \multicolumn{2}{|c|}{ Apoyos familiares } \\
\hline & Hombres & Mujeres & Hombres & Mujeres \\
\hline Cuernavaca & $\begin{array}{l}\text { Cuidado de los nietos: darles } \\
\text { de comer, recogerlos de la } \\
\text { escuela, arreglos domésticos }\end{array}$ & $\begin{array}{l}\text { Quehaceres domésticos, } \\
\text { barrer, cocinar y cuidar o } \\
\text { recoger a los nietos cuando } \\
\text { salen de la escuela }\end{array}$ & $\begin{array}{c}\text { Yernos, nueras, hijos e hijas, } \\
\text { nietos }\end{array}$ & $\begin{array}{l}\text { Yernos, nueras, hijos e hijas, } \\
\text { nietos }\end{array}$ \\
\hline Chilpancingo & $\begin{array}{l}\text { Lavar el patio, barrer, hacer } \\
\text { arreglos domésticos }\end{array}$ & Cuidar a los nietos & Cónyuge, hijos e hijas & Cónyuge, hijos e hijas \\
\hline Culiacán & $\begin{array}{c}\text { Proveen apoyo económico a } \\
\text { las hijas que son abandonadas } \\
\text { por sus maridos }\end{array}$ & $\begin{array}{l}\text { Cocinar, lavar, hacer los } \\
\text { quehaceres, cuidar de otros } \\
\text { enfermos, como sus esposos } \\
\text { y en algunos casos se dan } \\
\text { tiempo para poder visitar y } \\
\text { apoyar a otras personas más } \\
\text { envejecidas, más pobres o } \\
\text { más enfermas que ellas }\end{array}$ & $\begin{array}{c}\text { Cónyuges, cuando se tienen, } \\
\text { hijos e hijas }\end{array}$ & $\begin{array}{c}\text { Cónyuges, cuando se tienen, } \\
\text { hijos e hijas }\end{array}$ \\
\hline Guadalajara & Cuidar a los nietos & $\begin{array}{l}\text { Cuidan a otros enfermos o } \\
\text { menores de edad (nietos), } \\
\text { realizan la mayor parte del } \\
\text { trabajo doméstico y varias de } \\
\text { ellas desarrollan actividades } \\
\text { informales diversas para } \\
\text { poder salir adelante con los } \\
\text { gastos de la familia (venta } \\
\text { de productos). Cuidado de } \\
\text { los nietos, debido al empleo } \\
\text { materno de sus propias hijas } \\
\text { o nueras }\end{array}$ & $\begin{array}{c}\text { Cónyuge, cuando se tiene, } \\
\text { quien es considerada la más } \\
\text { importante, y los hijos e hijas. } \\
\text { Vecinos. La migración aparece } \\
\text { como un elemento importante } \\
\text { que media la solidez de las } \\
\text { redes }\end{array}$ & $\begin{array}{c}\text { Cónyuge (si está sano y/o si } \\
\text { puede seguir trabajando) y los } \\
\text { propios hijos e hijas }\end{array}$ \\
\hline
\end{tabular}

Fuente: elaboración propia con base en grupos focales con adultos mayores en Cuernavaca, Chilpancingo, Guadalajara y Culiacán, 2005.

ción económica y de salud de los adultos mayores, imponen a las familias necesidades específicas de apoyo, cuya satisfacción se encuentra mayoritariamente en la propia red familiar y vecinal, muy poco es lo que contribuyen otro tipo de actores como las iglesias o programas de gobierno.

Esta condición coincide con estudios que se han realizado en América Latina 15, donde se muestra que a pesar de todos los cambios en la estructura y dinámica que pueden haber llevado a la fragmentación de las redes familiares, los adultos mayores cuentan fundamentalmente con el apoyo de la familia para poder solventar sus problemas de acceso a comida y medicamentos, debido al debilitamiento de redes comunitarias. No obstante, en el futuro la capacidad de las familias para asumir ciertas responsabilidades, ante el apoyo que demandan los diferentes miembros, podría disminuir de forma impor- tante, a causa de tres aspectos fundamentales: la disminución del número de miembros de la familia y potenciales proveedores de apoyo por la baja de la fecundidad 31 ; el debilitamiento del esquema de cuidado fundamentalmente provisto por mujeres, por motivo de su creciente participación en el proceso económico-laboral 32 (Sánchez-Ayendez, 1994, citado por Guzmán et al. 18) y por último, la modificación del modelo de residencia, basado en la cohabitación de los adultos mayores con sus familias, como resultado de cambios en los patrones de nupcialidad y el aumento de las necesidades de esta población que demandará cada vez más recursos médicos costosos 33 .

Estos elementos deben estar presentes en el debate académico y político sobre la reorganización de los sistemas de protección social, tal como lo señala Aguirre 34 , en el que se defina la responsabilidad de los cuidados de los adultos 
mayores, ya que sin duda tienen un impacto importante frente a la necesidad de apoyo de este sector de la población.

Es importante mencionar que los resultados de este estudio también confirmaron que los adultos mayores que viven acompañados refieren una mejor experiencia de envejecimiento en comparación con quienes viven solos. La integración social de los adultos mayores juega un papel más importante que muchos de los riesgos tradicionalmente considerados para ellos: " $\mathrm{el}$ estar aislado supone un riesgo para la salud en magnitud comparable a los riesgos asociados al hábito de fumar, la presión arterial elevada y la obesidad" 15 (p. 76).

Este estudio identificó al igual que otros 18,35, que el apoyo brindado es bidireccional, es decir, los adultos mayores reciben apoyo por parte de hijos, vecinos o del cónyuge; pero al mismo tiempo ellos también proveen ayuda en la forma de cuidados hacia los nietos, trabajos domésticos o actividades laborales informales para conseguir ingresos para la casa. Es de resaltar la participación de los adultos mayores en el flujo de intercambios que caracteriza a los apoyos sociales que se dan en el marco de las redes sociales 36,37 , ya que con frecuencia se infravalora el importante papel que ellos juegan. Este intercambio depende de las condiciones de salud, funcionalidad y recursos de los adultos mayores, lo que dan depende de cómo estén y cómo se les percibe, pues aunque estén dispuestos a dar ayuda no siempre tienen las condiciones para brindarla, situación que acentúa en ellos sentimientos de depresión y tristeza.

Uno de los hallazgos principales de este estudio, tal como reportan varios autores 18,24,38, es que los tipos de apoyos identificados para estos ancianos empobrecidos de zonas urbanas son fundamentalmente de carácter material (alimentos, medicinas), económico (dinero o pago de consultas) e instrumental (traslado a consultas o tratamientos médicos). Muy escasamente se identificó el acompañamiento cotidiano y la inserción social en el grupo familiar o social (charla y apoyo emocional o cognitivo continuo), atención en un sentido amplio, como una necesidad humana fundamental.

Se resalta que el apoyo es diferenciado según el género, lo cual ha sido reportado por varios autores 15,38 (Derber, 1979, citado por Castro et al. ${ }^{39)}$. Los hombres reciben apoyo económico con mayor frecuencia y menos emocional; aún cuando en esta etapa de la vida varias de las necesidades expresadas se refieren a los sentimientos de soledad y abandono y al deseo de estar más acompañado. Por tanto, podría parecer que los actores sociales que prestan más apoyo lo hacen en términos de una acción momentánea y específica y menos como un proceso más duradero de atención que implica la convivencia cotidiana, lo cual, según varios autores (Derber, 1979, citado por Castro et al. 39), también está relacionado con la calidad de vida, el bienestar y la propia salud. Esto coincide con la discusión elaborada por Guzmán et al. 18, en el sentido de que durante años se consideró que si la persona tenía una red contaba necesariamente con apoyo, pero la investigación gerontológica ha desmentido esta idea al profundizar en el estudio del tipo de apoyo, frecuencia y duración y al explorar la relación con la satisfacción de necesidades básicas. En el mismo trabajo los autores proponen una interesante dimensión de análisis que identifica la percepción del apoyo recibido y la comparan con la percepción del apoyo que se ofrece, pues con frecuencia se identifica que los adultos mayores perciben que ellos aportan más de lo que reciben a cambio.

Situaciones como contar con seguridad social, dependiente de la situación laboral de los hijos o la posibilidad de conseguir alimentos, según si la vecina puede o no ofrecérselos, nos hablan de una conectividad inestable de los apoyos identificados (son esporádicos, puntuales y poco seguros). Los constantes temores expresados al respecto por parte de los adultos mayores, y su falta de control sobre ello, los hace aún más vulnerables en comparación con otros grupos de adultos mayores. Tal como afirman Liem \& Liem (1978), citados por Castro et al. 39 (p. 429): “el papel y la importancia del apoyo social y del control personal es complejo y varía según clase social y niveles de estrés".

De acuerdo con la afirmación de Pearlin 40, existen diferencias en el tipo de apoyos que pueden prestarse a los miembros de una red según la clase social, porque los bienes y el poder están distribuidos de manera desigual en la sociedad; en este sentido, la experiencia de envejecer es radicalmente diferente. En muchos países, la falta de cobertura de los servicios de salud y de planes de jubilación, así como la exclusión del mercado laboral formal, resaltan la existencia de un importante número de adultos mayores que no tiene acceso a mecanismos institucionales para satisfacer sus necesidades y que depende de su familia para la supervivencia 18,41. México está muy lejos de cubrir satisfactoriamente las necesidades de esta población, particularmente de los más vulnerables: empobrecidos y solos. En este sentido, es necesario incrementar las acciones gubernamentales, comunitarias y sociales para los adultos mayores que contribuyan a la creación de programas y políticas públicas que enfrenten las necesidades de salud, econó- 
micas, de servicios y aún de integración social y recreación, que con base en las proyecciones para el futuro se producirán de manera inminente. Resulta indispensable que en las políticas para el adulto mayor se considere la propuesta de la Organización Mundial de la Salud sobre el envejecimiento activo, cuyos pilares son la salud, la participación y la seguridad, como factores protectores frente a las enfermedades que discapacitan en la vejez 15 .

En la actualidad el reto para la sociedad es cambiar la forma como se vive la vejez, lo cual

\section{Resumen}

El objetivo del artículo fue analizar el apoyo social y las condiciones de vida de adultos mayores en zonas urbanas empobrecidas. Estudio cualitativo con 8 grupos focales, realizado en Guerrero, Jalisco, Morelos y Sinaloa, México, durante 2005-2006. Participaron 40 varones y 63 mujeres. El principal apoyo para la vida cotidiana y la condición de salud de los adultos mayores proviene de los familiares cercanos, en algunos casos de vecinos. Los apoyos de los adultos mayores son básicamente materiales y económicos, así como cierto tipo de acompañamiento y traslado a consultas médicas. El apoyo emocional es mínimo o inexistente, igual la compañía cotidiana y la integración en la vida familiar. Se identificó una ausencia significativa de apoyo por parte del gobierno o de organizaciones religiosas o civiles. La familia continúa siendo la fuente fundamental de apoyo. Es necesario incrementar las acciones gubernamentales para la población adulta mayor y transformar la idea de que las necesidades de esta población es una responsabilidad individual más que colectiva.

Ancianos; Pobreza; Condiciones Sociales; Apoyo Social necesariamente implica considerar algunos determinantes identificados en este estudio como la clase social y el género. El objetivo no es alcanzar más años de vida, sino lograr que los años que se vivan sean con calidad y dignidad, independientemente de ser hombre o mujer y de tener cierta posición social, lo cual nos lleva de nuevo a la reflexión del envejecimiento como una responsabilidad social, no como una situación individual que cada persona debe resolver según sus recursos sociales, culturales, emocionales o económicos.

\section{Colaboradores}

B. E. Pelcastre-Villafuerte contribuyó con el diseño del estudio, levantamiento de información, análisis de datos, propuesta del artículo, primera versión completa y revisión de las versiones subsecuentes. S. Treviño-Siller realizó el levantamiento y análisis de información, revisión crítica del artículo y versiones subsecuentes. T. González-Vázquez participó del diseño del estudio, análisis de información, revisión crítica del artículo y versiones subsecuentes. M. Márquez-Serrano participó del diseño del estudio, levantamiento de información, análisis de datos, revisión crítica del artículo y versiones subsecuentes.

\section{Agradecimientos}

Instituto Nacional de Desarrollo Social (INDESOL: CS09-I-VI-057-05). 


\section{Referencias}

1. Consejo Nacional de Población. Proyecciones de la población de México 2005-2050. http://www. conapo.gob.mx/00cifras/5.htm (accedido el 06/ Mar/2008).

2. Consejo Nacional de Población. Encuesta Nacional de la Dinámica Demográfica 2006 (ENADID). http://www.conapo.gob.mx/encuesta/Enadid/in dex.htm (accedido el 25/Mar/2009).

3. Partida V. Perspectivas del cambio demográfico. Monto y estructura de la población en el año 2000 y perspectivas en el 2050. DemoS 2001; (14):6-7.

4. Bolvitnik J. Conceptos y métodos para el estudio de la pobreza. Revista de Comercio Exterior 2003; 53:404-9.

5. Cáceres FC. Dimensiones sociales y relevantes para la prevención del VIH/SIDA en América Latina y el Caribe. In: Izazola JA, editor. El SIDA en América Latina y el Caribe: una visión multidisciplinaria. México DF: Fundación Mexicana para la Salud; 1999. p. 217-46.

6. Aparicio R. Transición demográfica y vulnerabilidad durante la vejez. In: Consejo Nacional de Población, editor. La situación demográfica de México. México DF: Consejo Nacional de Población; 2002. p. 155-68.

7. Wong R, Espinoza M, Palloni A. Adultos mayores mexicanos en contexto socioeconómico amplio: salud y envejecimiento. Salud Pública Méx 2007; 49:436-47.

8. Gallegos-Carrillo K, Mudgal J, Sánchez-García S, Wagner FA, Gallo JJ, Salmerón J, et al. Social networks and health-related quality of life: a population based study among older adults. Salud Pública Méx 2009; 51:6-13.

9. Puga D, Rosero-Bixby L, Glaser K, Castro T. Red social y salud del adulto mayor en perspectiva comparada: Costa Rica, España e Inglaterra. Población y Salud en Mesoamérica 2007; 5:1-21.

10. Curcio-Borrero CL. Soporte social informal, salud y funcionalidad en el anciano. Hacia la Promoción de la Salud 2008; 13:52-8.

11. Leite MT, Battisti IDE, Berlezi EM, Scheuer AI. Idosos residentes no meio urbano e sua rede de suporte familiar e social. Texto \& Contexto Enferm 2008; 17:250-7.

12. Rioseco HR, Quezada VM, Ducci VME, Torres HM. Cambio en las redes sociales de adultos mayores beneficiarios de programas de vivienda social en Chile. Rev Panam Salud Pública 2008; 23:147-53.

13. Salinas A, Manrique B, Téllez-Rojo MM. Redes de apoyo social en la vejez: adultos mayores beneficiarios del componente para Adultos Mayores del Programa Oportunidades. In: III Congreso de la Asociación Latinoamericana de Población, ALAP, realizado en Córdoba -Argentina, del 24 al 26 de septiembre de 2008. http://www.alapop. org/2009/images/DOCSFINAIS_PDF/ALAP_2008_ FINAL_281.pdf.

14. Grijalva-Monteverde G, Zúñiga-Elizalde M, ZupoJiménez MJ. Adultas y adultos mayores en Sonora: ¿dependientes, autosuficientes o proveedores? Región y Sociedad 2007; XIX número especial:117-45.
15. Peláez MB, Rodríguez-Wong L. Vejez y recursos sociales en América Latina. Humanitas: Humanidades Médicas 2004; (1):73-83.

16. Centro Latinoamericano y Caribeño de Demografía-División de Población, Comisión Económica para América Latina y el Caribe. Presentación. Notas Poblac 2002; XXIX:7-9.

17. Huenchuan S, Guzmán JM. Seguridad económica y pobreza en la vejez: tensiones, expresiones y desafíos para el diseño de políticas. Notas de Población CEPAL s/f; (83):99-125.

18. Guzmán JM, Huenchuan S, Montes de OcaV. Redes de apoyo social de las personas mayores: marco conceptual. http://www.eclac.cl/publicaciones/ Poblacion/3/LCG2213P/lclg2213_p2.pdf (accedido el 17/Abr/2006).

19. Cassel J. Psychosocial processes and stress: theoretical formulation. Int J Health Serv 1974; 4: 471-82.

20. Cobb S. Social support as a moderator of life stress. Psychosom Med 1976; 38:300-14.

21. Thoits PA. Life stress, social support and psychological vulnerability: epidemiological considerations. J Community Psychol 1982; 10:341-62.

22. Cohen S, Wills TA. Stress, social support and the buffering hypothesis: a theoretical analysis. Psychol Bull 1985; 98:10-357.

23. Barrón A, Sánchez-Moreno R. Estructura social, apoyo social y salud mental. Psicothema 2001; 13:17-23.

24. Matud P, Carballeira M, López M, Marrero R, Ibáñez I. Apoyo social y salud: un análisis de género. Salud Mental 2002; 25:32-7.

25. Tuirán R. Estructura familiar y trayectorias de vida en México. In: Gomes C, compilador. Procesos sociales, población y familia. México DF: Facultad Latinoamericana de Ciencias Sociales/Porrúa; 2001. p. 23-65.

26. García H. Sistemas de soporte a la vejez: apoyos formales e informales en el área metropolitana de Monterrey. In: Hernández H, Menkes C, editores. La población mexicana a finales del siglo XX. México DF: Sociedad Mexicana de Demografía/ Centro Regional de Investigaciones Multidisciplinaria, Universidad Nacional Autónoma de México; 1998. p. 399-416.

27. Morgan D. When to use focus groups and why. In: Morgan LD, editor. Successful focus groups. London: Sage Focus Editions; 1997. p. 3-19.

28. Streubert HJ, Carpenter DR. Phenomenological research approach. In: Streubert HJ, Carpenter DR, editors. Qualitative research in nursing. Advancing the humanistic imperative. New York: J. B. Lippincott Co.; 1995. p. 29-49.

29. Fairclough N. Discourse and social change. Cambridge: Polity Press; 1992.

30. Íñiguez L, editor. Análisis del discurso. Manual para las ciencias sociales. 2a Ed. Barcelona: Universitat Oberta de Catalunya; 2006.

31. Enríquez R. Dinámica de las redes sociales y de apoyo emocional en hogares pobres urbanos: el caso de México. http://136.142.158.105/Lasa2000/ EnriquezRosas.PDF (accedido el 17/Abr/2006). 
32. Benítez R. La población y el desarrollo en México: la desigualdad social y sus consecuencias demográficas. In: Jiménez R, Minujin A, coordinadores. Los factores del cambio demográfico en México. México DF: Siglo XXI/Instituto de Investigaciones Sociales, Universidad Nacional Autónoma de México; 1984. p. 379-415.

33. Pérez J, Brenes G. Una transición en edades avanzadas: cambios en los arreglos residenciales de adultos mayores en siete ciudades latinoamericanas. Estudios Demográficos y Urbanos 2006; 21:625-61.

34. Aguirre R. Las familias como proveedoras de servicios de cuidados. In: Astelarra J, coordinador. Género y cohesión social. Madrid: Fundación Carolina; 2007. p. 83-93. (Documento de Trabajo, 16).

35. Montes de Oca V. Envejecimiento y protección familiar. Límites y potencialidades del apoyo al interior del hogar. In: Ariza M, Oliveira O, coordinadores. Imágenes de la familia en el cambio de siglo. México DF: Instituto de Investigaciones Sociales, Universidad Nacional Autónoma de México; 2004. p. 519-63.

36. Lomnitz L. Cómo sobreviven los marginados. México DF: Siglo XXI; 1975.
37. Muchinik E. Las redes sociales de apoyo. Rev Argent Clín Psicol 1990; II:174-81.

38. Ham-Chande R, Ibáñez E, Torres AL. Redes de apoyo y arreglos de domicilio de las personas en edades avanzadas en la Ciudad de México. Notas Poblac 2003; 77:71-102.

39. Castro R, Campero L, Hernández B. La investigación sobre apoyo social en salud: situación actual y nuevos desafíos. Rev. Saúde Pública 1997; 31: 425-35.

40. Pearlin LI. Social structure and processes of social support. In: Cohen S, Syme SL, editors. Social support and health. New York: Academic Press; 1985. p. $43-60$.

41. Sunkel G. El papel de la familia en la protección social en América Latina. In: Astelarra J, coordinador. Género y cohesión social. Madrid: Fundación Carolina; 2007. p. 95-106. (Documento de Trabajo, 16).

Recibido el 03/Feb/2010

Versión final presentada el 01/Dic/2010

Aprobado el 03/Ene/2011 